\title{
Multilinguales
}

Pratiques littéraires, linguistiques, pédagogiques, didactiques et médiations culturelles contemporaines

\section{Rôle de la littérature dans le développement de la compétence communicationnelle en cours d'allemand au Burkina Faso}

The Role of Literature in the Acquisition of the Communicative Competence

during a German Course in Burkina Faso

Jean-Claude Bationo

\section{(2) OpenEdition}

Journals

\section{Édition électronique}

URL : https://journals.openedition.org/multilinguales/3056

DOI : 10.4000/multilinguales.3056

ISSN : 2335-1853

Éditeur

Université Abderrahmane Mira - Bejaia

\section{Édition imprimée}

Date de publication : 1 juin 2013

Pagination : $69-79$

ISSN : 2335-1535

\section{Référence électronique}

Jean-Claude Bationo, «Rôle de la littérature dans le développement de la compétence

communicationnelle en cours d'allemand au Burkina Faso », Multilinguales [En ligne], 1 | 2013, mis en ligne le 01 juin 2013, consulté le 30 juin 2021. URL : http://journals.openedition.org/multilinguales/ 3056 ; DOI : https://doi.org/10.4000/multilinguales.3056

Ce document a été généré automatiquement le 30 juin 2021.

\section{(c) $($ ) $\odot \odot$}

Multilinguales est mise à disposition selon les termes de la Licence Creative Commons Attribution Pas d'Utilisation Commerciale - Pas de Modification 4.0 International 


\title{
Rôle de la littérature dans le développement de la compétence communicationnelle en cours d'allemand au Burkina Faso
}

\author{
The Role of Literature in the Acquisition of the Communicative Competence \\ during a German Course in Burkina Faso
}

Jean-Claude Bationo

1 Dans les diverses situations de la vie courante, le but primordial de la conversation est la compréhension mutuelle des interlocuteurs, la transmission des idées et l'élargissement de l'horizon de leurs connaissances. A travers la conversation, nous extériorisons nos sentiments, nos émotions, nos impressions, nos jugements qu'ils soient de valeur ou de réalité, de sorte à les partager avec les autres, à transmettre des informations et à en recevoir, à argumenter, à nous défendre, etc. En un mot, le dialogue permet de nouer des contacts avec les autres. L'apprenant de l'allemand, au Burkina Faso, devra maitriser l'allemand oral non seulement pour pouvoir « communiquer » dans les situations courantes, mais aussi pour comprendre, dans cette langue, les annonces publiques, les émissions radiophoniques et télévisuelles, les conférences, les spectacles, les représentations théâtrales, etc. Il sera également apte à s'engager dans des clubs d'allemand, ou à exercer le métier d'enseignant d'allemand dans les lycées et collèges ou celui d'agent de tourisme et d'hôtellerie ${ }^{1}$, s'il opte pour l'une ou l'autre de ces professions.

2 Il est donc indispensable de préparer les élèves à optimaliser leurs échanges communicatifs. Ce souci a conduit à l'introduction des actes de communication ${ }^{2}$ dans le programme de l'enseignement de l'allemand au Burkina Faso: saluer, se présenter, présenter quelqu'un, donner son âge et demander celui de son interlocuteur, parler ou répondre au téléphone, exprimer sa joie ou sa tristesse, etc., (Mulo, 1999). 
On peut alors prôner une didactique de la conversation dont le but est de développer, chez l'apprenant, une compétence en expression orale, voire une «maîtrise de la situation de communication orale » (Dolz et Schneuwly, 1998 : 91). La langue allemande, en effet, «ne s'acquiert pas par l'apprentissage des structures grammaticales et du lexique, mais doit être apprise à part entière en ayant recours à la science et à l'éducation de la conversation $»^{3}$ (Forster, $1997: 26$ ).

4 Nous chercherons à montrer dans quelle mesure la littérature peut contribuer à améliorer cette compétence en communication orale, aussi bien à l'école que dans d'autres contextes. Notre thèse, pour atteindre cet objectif, dans un contexte où la plupart des apprenants n'entendent et ne parlent l'allemand qu'en classe, consiste à développer la capacité en "conversation littéraire $»^{4}$. "Converser » en allemand, en cours d'allemand, est une stratégie d'apprentissage qui consiste à "re-créer » les conditions d'un bain linguistique pour des apprenants francophones (cf. Bationo $2007 \mathrm{a}$, Kangni, 2007), comme nous le montrerons ici même.

L'objectif de la présente contribution est de montrer l'intérêt didactique d'utiliser davantage la littérature en cours d'allemand langue étrangère.

Dans notre démarche, nous exposerons d'abord les objectifs du cours d'allemand au Burkina Faso. Ensuite, en nous référant aux résultats de certaines investigations de terrain, nous montrerons que la communication orale, qui est l'objectif principal du cours d'allemand, est aussi l'une de ses faiblesses. Enfin, nous introduirons l'idée que les potentialités linguistiques, culturelles et pédagogiques de la littérature permettent de contribuer au développement de la compétence communicationnelle ${ }^{5}$ des apprenants d'allemand. Pour cela, il est nécessaire, pour nous, d'esquisser l'histoire de l'intégration de la littérature dans l'enseignement de l'allemand langue étrangère, puis de discuter de l'importance de la conversation littéraire et, enfin, d'illustrer cette réflexion théorique par l'ébauche d'une proposition didactique autour de la légende Die Loreley en classe d'allemand au Burkina Faso.

\section{Les objectifs du cours d'allemand}

7 L'enseignement et l'apprentissage des langues vivantes, de façon générale, visent quatre compétences linguistiques, à savoir la compréhension de l'oral et la compréhension de l'écrit, l'expression orale et l'expression écrite.

8 Le système scolaire burkinabé assigne les mêmes objectifs à l'enseignement des langues :

Désormais l'enseignement des langues vivantes, allemand, anglais, arabe, etc., devra viser essentiellement entre la connaissance et une certaine familiarité avec la civilisation et la littérature des peuples dont la langue fait l'objet de l'étude, l'acquisition d'une compétence linguistique certaine. Ce qui veut dire que l'objectif de cet enseignement doit être non seulement la maîtrise des règles de la langue, mais aussi, et surtout le pouvoir de communiquer normalement dans ladite langue. Cet enseignement devra avoir un caractère pratique et immédiatement profitable. Tout élève arrivant en fin de cycle devra pouvoir soutenir une conversation normale avec un locuteur natif de son niveau d'étude. (Circulaire $n^{\circ} 105 /$ M.E.N.A.C./C.E.S.D., du $1^{\text {er }}$ décembre 1983)

D'après cette circulaire, les objectifs de l'enseignement de l'allemand, tout comme les autres langues étrangères, consistent en la pratique de la langue allemande, c'est-à-dire 
la communication, d'une part, et d'autre part, en l'acquisition des valeurs socioculturelles. La dernière phrase du texte officiel ci-dessus cité exige un haut niveau de compétence linguistique chez l'apprenant burkinabé. Autrement dit, le cours d'allemand a pour rôle de produire des locuteurs burkinabés capables de bien converser avec des interlocuteurs allemands.

10 Selon le Cadre Européen Commun de Référence pour les Langues, l'utilisateur de langue étrangère de niveau $\mathrm{B} 2$ « peut communiquer avec un niveau d'aisance et de spontanéité tel qu'une interaction soutenue avec les locuteurs natifs soit tout à fait possible sans entraîner de tension d'une part ni d'autre » (CECR, 2005 : 61). Il peut aussi correspondre au niveau B1 (ibid.). Cependant, dans la réalité, les apprenants germanistes n'ont pas ces niveaux de B1 ni de B2. Le niveau d'expression orale dont parle le texte officiel est un objectif à atteindre.

11 Par ailleurs, les enquêtes menées sur l'enseignement et l'apprentissage de l'allemand au Burkina (cf. Bationo, 2007 a : 58-59) révèlent que le niveau de la compétence en expression orale est en deçà des attentes des enseignants et des apprenants : 76,09\% des élèves ne sont pas satisfaits de leur niveau en expression écrite et orale ${ }^{6}$. Cette position est confirmée par $83,33 \%$ des conseillers pédagogiques. De plus, $8,82 \%$ des professeurs estiment que ce niveau est faible ${ }^{7}$. Cette insatisfaction s'est confirmée par les résultats des enquêtes menées en 2011 par l'inspecteur d'allemand Ouattara (2011: 35). Les objectifs du cours d'allemand relatifs à la compétence en communication orale, au Burkina Faso, sont donc loin d'être atteints, d'où l'intérêt de la présente réflexion.

12 Le cours d'allemand au Burkina Faso, comme dans bien d'autres pays africains francophones au sud du Sahara (cf. Kangni, 2007; Mbia, 1998, Mulo, 1999; Ngatcha, 1991, 2002), s'appuie le plus souvent, et pour l'essentiel, sur des dialogues, des textes fabriqués, des textes d'information, etc. La lecture rapide et univoque d'unités lexicales, l'acquisition des moyens de locution pour la réalisation d'intentions de communication, l'apprentissage et l'exercice de nouvelles structures de grammaire, etc. sont quelques objectifs visés par l'emploi de ces textes (Bationo, 2007 a). Ceux-ci ne favorisent pas le développement de la compétence de communication dans la mesure où ils donnent aux élèves des informations univoques, voire monosémiques (Eismann \&Thurmair, 1993 : 377).

13 L'enseignant attend des élèves qu'ils répondent simplement et succinctement aux questions portant sur ces textes lus en classe, et ce, aux fins de s'assurer de leur compréhension. Ainsi conçu, le cours d'allemand est un cours de conversation dialectique, c'est-à-dire une forme particulière de communication destinée à transmettre et à expliquer des règles de grammaire, de vocabulaire et des locutions. Si ce cours est en partie basé sur la communication orale, il faudrait former les élèves qui y participent en développant chez eux des compétences en expression orale. L'une des insuffisances majeures de l'acquisition de la langue allemande réside dans le fait qu'elle n'est pas pratiquée en contexte extrascolaire.

14 En effet, les résultats de travaux empiriques de 2004 (cf. Bationo, 2007 a), confortés en 2011 (cf. Ouattara) ont révélé que ce qui a été appris par la participation au cours a peu de valeur d'usage dans la réalité, car les élèves n'arrivent pas à communiquer entre eux ou avec d'autres locuteurs germanophones en dehors de la classe. Du reste, cette forme de communication en classe, dominée par la figure du professeur, est problématique : sa structure principale a une dimension verticale (professeur-élève) et les apprenants ne prennent la parole que lorsqu'ils doivent poser des questions ou y répondre. Ces 
observations valent également pour le cours d'allemand au Burkina, comme relevé dans un mémoire de fin de formation d'un inspecteur d'allemand :

L'allemand se présente pour beaucoup d'élèves, comme une langue parlée seulement en classe, plus exactement pendant le cours... L'expérience montre qu'en classe, « parler » se réduit le plus souvent, soit à répondre à une question (si on a la chance d'être interrogé), soit à lire un passage en allemand, mais rarement à engager une vraie conversation, quelque chaotique qu'elle soit, avec un partenaire en classe, encore moins ailleurs. (Ouedraogo, $2004: 15$ )

Tout le cours est donc dirigé par l'enseignant qui définit le cadre dans lequel les participants doivent s'exprimer. Grünwaldt (1998:65) fait remarquer à ce sujet que cette méthode "magistrocentrée " permet à peine aux élèves de prendre l'initiative pour discuter et convaincre leurs partenaires à l'aide d'arguments. Au Burkina Faso, même des étudiants de la langue allemande éprouvent encore des difficultés à parler couramment l'allemand bien qu'ils en maitrisent la grammaire et le vocabulaire et bien qu'ils soient capables de le comprendre et de l'écrire correctement. Puisque les objectifs de la compétence en expression orale sont difficiles à atteindre (Bationo, 2009 : 369), les méthodes de l'enseignement de l'allemand devraient être remises en question.

Ces faiblesses en communication orale en cours d'allemand hors des pays germanophones ne concernent pas seulement l'école burkinabé, mais également celle de bien d'autres pays dans lesquels l'allemand est enseigné comme première ou deuxième langue vivante ${ }^{8}$. Selon Forster l'expression orale ne s'acquiert pas par l'apprentissage des structures grammaticales et du lexique, mais par l'enseignement/ apprentissage de la conversation (1997: 26). Elle a besoin, pour se réaliser, d'échanges d'idées et d'opinions (Ritz-Fröhlich, $1982: 14$ ).

17 La littérature n'offrirait-elle pas cette opportunité d'échanges d'idées et d'opinions sur les discours des personnages ou sur les points de vue des auteurs?

\section{La littérature dans l'acquisition de la compétence communicationnelle}

18 Avant de montrer l'importance de la littérature dans l'acquisition de la compétence en communication orale, il n'est pas inopportun d'évoquer d'abord, de manière même sommaire, quelques résultats d'une enquête que nous avons menée, en 2004, auprès de 277 acteurs de l'enseignement et de l'apprentissage de l'allemand au Burkina Faso (cf. Bationo, 2007 a : 48-110).

19 Cette étude portait sur l'articulation littérature-acquisition de la compétence en expression orale. A la question «quelle est l'importance de la littérature dans l'acquisition de la compétence en expression orale ", voici les réponses obtenues : pour $25 \%$ des élève-professeurs et $14,70 \%$ des professeurs, les textes littéraires jouent un rôle capital; pour $44,85 \%$ des élèves, $62,50 \%$ des élève-professeurs, $41,17 \%$ des professeurs et $66,66 \%$ des conseillers pédagogiques de l'enseignement secondaire, la littérature joue un rôle relativement important ; pour 22,92 \% des élèves, $12,50 \%$ des élève-professeurs, $32,35 \%$ des professeurs et $33,33 \%$ des conseillers pédagogiques de l'enseignement secondaire, les textes littéraires sont partiellement importants 9 . 
C'est à partir de ces résultats que nous avons pensé proposer l'intégration de la littérature au cours d'allemand, comme moyen d'optimalisation de la compétence orale en langue allemande au Burkina Faso.

Rappelons que l'introduction de la littérature dans l'enseignement des langues étrangères remonte, selon Neuner et Hunfeld (2002: 7), au 19e siècle, et s'inscrivait dans le cadre des objectifs de l'enseignement au lycée qui consistaient notamment à donner à l'élève une culture générale. On avait recouru à la «méthode grammairetraduction ", à laquelle on a reproché par la suite d'enseigner une langue vivante avec des moyens et des règles d'une langue morte (Viëtor, 1882).

Le rejet de cette méthode, à travers celui de l'apprentissage par cœur des règles de grammaire, des mots et de leur traduction, a eu pour conséquence l'adoption de la méthode directe qui a privilégié «la maîtrise de la langue orale active » (Neuner et Hunfeld, 2002 : 34), la pratique de la communication orale, comme le souligne Bender J. (1979) :

L'objectif du cours dans les nouvelles langues a connu un changement dans toutes les grandes écoles où l'emploi pratique de l'oral et de l'écrit de la langue étrangère [...] est partout relégué au premier plan, la grammaire n'est qu'un moyen pour atteindre cet objectif $»^{10}$. (16)

Cette méthode directe mettait l'accent sur les compétences linguistiques ("comprendre» et "parler») et exigeait que l'apprenant fasse fi de sa langue maternelle. Il lui fallait penser, imaginer, comprendre et parler directement dans la langue étrangère, sans recourir à la traduction, contrairement à la méthode précédente. La fonction de la littérature n'apparaissait pas encore clairement. Mais, par rapport à la nouvelle orientation de la finalité de l'enseignement des langues étrangères, les textes littéraires servaient de base pour les exercices d'expression orale.

De la méthode directe, il en est résulté les méthodes "audio-linguale» et "audiovisuelle» qui ont privilégié les compétences en compréhension auditive et en expression orale en s'appuyant sur des situations de la vie quotidienne. Dans la méthode audio-linguale, la littérature a été supprimée de l'enseignement et de l'apprentissage des langues étrangères.

5 Ce grand silence sur la didactique de la littérature a duré jusqu'aux années 1970 au cours desquelles les discussions sur la didactique des langues étrangères, dans une perspective pragmatique, ont permis l'orientation de l'enseignement/apprentissage vers la communication quotidienne et une focalisation sur la pédagogie de la transmission des connaissances culturelles (Neuner et Hunfeld, 2002 : 61). Ainsi, dans les années 1990, l'importance de la didactique de la littérature est revenue au centre des préoccupations pédagogiques de l'enseignement des langues étrangères.

Dans cette phase d'approche communicative, il s'est développé le concept de " pragmatique fonctionnelle ", emprunté à la linguistique pragmatique qui considère la langue comme un aspect du comportement humain (cf. Searle, 1969), et qui en met l'accent sur les intentions de parole. Cela fait passer ainsi au premier plan la pratique et la facilitation de la communication. Ce courant de la didactique communicative, qui a négligé la fonction de la littérature en cours des langues étrangères, prônait un enseignement qui reposait sur les situations communicatives relatives aux réalités de la langue cible à imiter. Il a résulté de cette stratégie, un nouvel objectif pour l'enseignement des langues étrangères: la découverte de la culture. Connaître sa propre culture et celle de l'Autre par l'apprentissage de la langue étrangère. Les 
intérêts des apprenants sont alors pris en compte dans le choix des textes littéraires (Pfäfflin, $2010: 28$ ).

\section{La conversation littéraire}

La «conversation littéraire» est définie par Christ et al. (1993: 25) comme des discussions ou entretiens en cours de littérature ou sur la littérature. L'un des meilleurs catalyseurs de discussions est, de notre point de vue, le texte littéraire, car celui-ci présente le plus souvent un message codé que chaque lecteur peut interpréter subjectivement, selon ses expériences et ses connaissances. Chaque lecteur peut avoir son point de vue sur le contenu d'un texte littéraire, d'où son caractère polysémique, polémique et labyrinthique ${ }^{11}$. Il n'est donc pas seulement un véhicule de savoirs, mais aussi un médium qui crée une dynamique interactive au sein de la classe.

En cours de littérature, des conversations et des discussions peuvent être initiées autour de thèmes en rapport avec la vie quotidienne des élèves (cf. Bationo, $2010 \mathrm{~b}$ : 114-116). A titre d'illustration, nous pouvons citer le thème du mariage forcé et du mariage précoce dans le sketch Die Überraschung (cf. Mbaye et al., $1998: 73$ ), les thèmes du chômage, de l'exode rural, de la ville, etc. dans le texte Kambara (cf. Mbaye et al., 1998 : 20), de la violence scolaire dans le texte Die Sache mit Christoph (cf. Mbaye et al., op.cit.: 32). L'ensemble des différentes interprétations de ce type de textes, les différents points de vue qu'ils suscitent contribueraient ainsi à un élargissement de l'horizon culturel de l'élève.

L'une des conditions pour la réalisation d'une conversation littéraire est le choix du texte littéraire. Il doit correspondre à l'âge et au niveau d'apprentissage de l'apprenant pour pouvoir constituer un levier de la conversation. Les textes doivent être non seulement intéressants, mais également accessibles aussi bien au niveau du contenu qu'à celui de la langue (cf. Bationo, $2010 \mathrm{a}: 142$ ).

30 De plus, selon Spinner (1987: 186), le comportement du professeur au cours de conversation littéraire a une grande influence sur la qualité de la prestation. Pour gérer la conversation en classe, il propose trois formules que le professeur peut suivre pour atteindre ses objectifs :

- il ne doit pas «mener l'élève à la lisière, mais plutôt lui donner des impulsions qui conduisent au développement d'une ébauche de compréhension »;

- Il doit présenter le cours de littérature de telle sorte qu'il y ait une alternance entre l'abstrait et le concret; Spinner (op.cit) explique que le texte littéraire perd sa force esthétique lorsque le professeur se met à développer des notions générales ;

- Il est conseillé au professeur de ne pas mettre très tôt les résultats des discussions au tableau afin que les élèves puissent continuer à interpréter et discuter entre eux sans être influencés par l'enseignant.

Merkelbach $(1998,: 80)$ est d'avis que le professeur doit se retenir de donner son interprétation personnelle pendant la conversation littéraire, et jouer le rôle de modérateur de la conversation. Il propose aussi que le professeur donne quelques informations sur le contexte du texte pour une meilleure compréhension du texte et qu'il introduise le thème de discussion. Selon Göldner (1971: 302), l'enseignant doit créer une atmosphère de confiance autour de la conversation. 
Mbaye et al., 1998 : 48-49), Der Mäuseturm von Bingen (cf. Hartenburg, J. et al., 1992

68) peuvent par exemple être suivis d'exercices écrits dans lesquels il serait demandé aux élèves d'imaginer les scènes. Selon l'IPAM (1996) : "l'étude de l'image permet sur le plan pédagogique, d'éduquer le regard, d'enrichir la sensibilité, de stimuler l'imagination, d'éveiller l'esprit critique et de développer l'expression » (55). littéraire, peut donc servir de tremplin pour mieux appréhender le contenu de ce texte. Avant d'aborder la légende Die Loreley dans le manuel scolaire d'allemand IHR und WIR II, (cf. Hartenburg, 1992 : 62) par exemple, il est souhaitable de commencer le cours en demandant aux élèves de décrire oralement l'image qui illustre le texte. C'est en quelque sorte "une mise en appétit » pour mieux préparer l'élève à « déguster » le 
texte de la légende. On peut aussi partir du texte de cette légende pour demander aux élèves de le prolonger avec leurs propres textes.

L'objectif de tels exercices est de stimuler la créativité de l'élève pour lui faire prendre conscience de sa capacité de production à l'écrit et à l'oral.

Ainsi, la présente réflexion aborde la contribution de la littérature dans l'acquisition de la compétence communicationnelle. De par ses potentialités polysémiques, polémiques et esthétiques, le texte littéraire bien choisi intéresse l'apprenant, le provoque, l'amuse, le distrait, le fait réfléchir sur lui-même, sur son environnement et l'amène enfin à la discussion, qualifiée de conversation littéraire. Il s'agit d'exploiter ces potentialités de la littérature pour développer le goût et la motivation de parler l'allemand devant les autres et avec eux en continu et en interaction. L'interaction entre élèves et enseignant et surtout entre élèves, en cours d'allemand, ne peut atteindre les objectifs assignés à l'expression orale et aux actes de communication en cours de langue que si l'enseignant joue le rôle de modérateur. Etant tous des «interlocuteurs » potentiels du texte littéraire, les élèves aussi (et surtout) peuvent s'exprimer sur l'histoire narrée, les thèmes, les personnages, etc. (Bationo, 2011); d'où le statut spécifique de la littérature comme support privilégié pour l'enseignement de l'allemand langue étrangère en général et pour l'acquisition de la compétence communicationnelle dans cette langue en particulier.

\section{BIBLIOGRAPHIE}

ALLHOFF, D.-W., Förderung mündlicher Kommunikation. Durch Therapie, Unterricht und Kunst. München, Ernst Reinhardt Verlag, 2001.

BATIONO, J. - C., Literaturvermittlung im Deutschunterricht in Burkina Faso. Stellenwert und Funktion literarischer Texte im Regionallehrwerk IHR und WIR, Teil I. Frankfurt am Main : Peter Lang, 2007 a.

BATIONO, J. - C., Literaturvermittlung im Deutschunterricht in Burkina Faso : Die Dissertation begleitende literarische Textauszüge zum interkulturellen Lernen im Unterricht Deutsch als Fremdsprache. Teil 2. Bern, Berlin, Brüssel, Frankfurt am Main : Peter Lang Verlag, 2007 b.

BATIONO, J. - C., « Verknüpfung von Grammatik- und Literaturdidaktik in Burkina Faso : Der Stellenwert literarischer Texte in einer Kulturkontrastiven Grammatik », in Götze, Lutz/Patricia Mueller-Liu/Salifou Traoré (éd.), Kulturkontrastive Grammatik - Konzepte und Methoden, Bern, Berlin, Brüssel, Frankfurt a.M., New York, Oxford, Wien, Peter Lang Verlag, 2009, pp. 243-251.

BATIONO, J. - C., « Le filtre des textes littéraires en cours d'allemand langue étrangère en Afrique francophone subsaharienne », Cahiers du cerle shs tome xxv 36, 2010 a, pp. 137-152.

BATIONO, J. - C., « L'usage des textes littéraires dans les manuels scolaires d'allemand en France et au Burkina Faso », Annales de l'Université de Ouagadougou, série A, vol. 011, décembre 2010 b, pp. 105-134. 
BATIONO, J. - C., « Rôle du manuel scolaire français dans la promotion de la littérature burkinabè écrite », Science et technique, vol. 26, n² 2, 2010 c, pp. 83-99.

BATIONO, J. - C., « Literatur und DaF-Unterricht : Zur Relevanz der Figuren im Literaturunterricht », Zielsprache Deutsch 38, 2., 2011, pp. 37-55.

BATIONO, J. - C., Introduction à la didactique de la littérature allemande, Ouagadougou Presses Universitaires de Ouagadougou, 2012.

BENDER, J., Zum gegenwärtigen Stand der Diskussion um Sprachwissenschaft und Sprachunterricht, Diesterweg : Frankfurt/Main, 1979.

CHRIST, H. et al., « In der Schule literarische Gespräche führen », Jahrbuch der Deutschdidaktik, Tübingen, 1993.

Cadre européen commun de référence pour les langues, apprendre, enseigner, évaluer, Paris, Conseil de l'Europe/Les Editions Didier, 2005.

DOLZ, J. et SCHNEUWLY, B., Pour un enseignement de l'oral. Initiation aux genres formels à l'école, Paris, ESF, 1998.

EISMANN, V. et THURMAIR, M., Literatur für Lerner. « Ein didaktisches Konzept für den Anfangsunterricht Deutsch als Fremdsprache", in Alois Wierlacher u. a. (Hrsg), Jahrbuch Deutsch als Fremdsprache, München, iudicium Verlag, Band 19, S., pp. 373-389, 1993.

FORSTER, R., Mündliche Kommunikation in Deutsch als Fremdsprache : Gespräch und Rede, St Ingbert, Röhrig Universitätsverlag, 1997.

GÖLDNER, H.-D., Planmäßige Pflege des Unterrichtsgesprächs, Blätter zur Lehrerfortbildung, 23, 1971, pp. 298-308.

GRÜNWALDT, H. J., « Zur Didaktik und Methodik mündlicher Kommunikations-Übungen », Der Deutschunterricht Velber 50, H.1, 1998, pp. 65-73.

HARTENBURG, J. et al., IHR und WIR. Text- UND ÜBUNGS-BUCH 2. Hamburg : Buchverlag Otto Heinevetter, 1992.

HUMIERE, C., « Sur le modèle du labyrinthe, lorsque la littérature privilégie le jeu », Amaltea Revista de mitocritica, vol 1, 2009, pp. 133-144.

IPAM, Enseigner le Français au collège et au lycée, Paris, EDICEF, 1996.

KANGNI, J. T., Fremdsprache Deutsch in Togo und ihre Funktion im togolesischen Erziehungsund Bildungssystem, Freiburg, Pädagogische Hochschule, 2007.

KAST, Bernd, Jugendliteratur im kommunikativen Deutschunterricht, Berlin und München, Langenscheidt KG, 1985.

MBAYE, D. et al., IHR und WIR. TEXT- UND ÜBUNGSBUCH 3. Hamburg, Buchverlag Otto Heinevetter, 1998.

MBIA, C.M.R., DaF - Unterricht in Afrika. Chancen Grenzen Möglichkeiten. Am Beispiel Kameruns, Freiburg, Pädagogische Hochschule, 1998.

MERKELBACH, V., « Über literarische Texte sprechen. Mündliche Kommunikation im Literaturunterricht », Der Deutschunterricht - Velber 50 H. 1, 1998, pp. 74-82.

MULO FARENKIA, B., Sprechaktkompetenz als Lernziel. Zur Didaktik einer kommunikativen Grammatik im Fach Deutsch als Fremdsprache, Frankfurt am Main : Peter Lang, 1999. 
NEUNER, G. und HUNFELD, H., Methoden des fremdsprachlichen Deutschunterrichts. Eine Einführung, Fernstudieneinheit 4, Berlin, München, Wien, New-York, Langenscheidt, 2002.

NGATCHA, A., Inhalte und Methoden des Deutschunterrichts an Kameruner Sekundarschulen. Bestandsaufnahme und zukünftige Möglichkeiten der interkulturellen Kommunikation, Ammersbek bei Hamburg, Verlag an der Lottbek, 1991.

NGATCHA, A., Der Deutschunterricht in Kamerun als Erbe des Kolonialismus und seine Funktion in der postkolonialen Ära, F/M. u.a : Peter Lang, 2002.

OUATTARA, F., Die Sprechpraxis im Deutschunterricht in Burkina Faso : Beitrag zur Förderung der Sprechfertigkeit in der Oberstufe, mémoire de fin de formation aux fonctions d'inspecteur de l'enseignement secondaire, option, allemand, Ecole Normale Supérieure de l'Université de Koudougou, version soutenue, 2011.

OUEDRAOGO, D., Evaluation des méthodes d'enseignement/apprentissage de l'allemand dans les établissements secondaires burkinabé : quelles stratégies pour l'amélioration de la compétence communicationnelle des apprenants d'allemand au Burkina Faso ? Mémoire de fin de formation aux fonctions d'inspecteur de l'enseignement secondaire, option allemand, Ecole Normale Supérieure de Koudougou, 2004

PFÄFFLIN, S., Auswahlkriterien für Gegenwartsliteratur im Deutschunterricht. Baltmannsweiler, Schneider Verlag Hohengehren, 2010.

RITZ-FRÖHLICH, G., Das Gespräch im Unterricht. Anleitung -Phasen- Verlaufsformen, Bad Heilbrunn, Klinkhardt, 1982.

SEARLE, J. R., Speech acts, Cambridge, University Press, 1969.

SPINNER, K. H., « Zur Rolle des Lehrers im Unterrichtsgespräch », in Sprache und Gefühle, Paderborn, Ferdinand Schöningh, 1987.

TANGER, G., « Muß der Sprachunterricht umkehren ? Ein Beitrag zur neusprachlichen Reformbewegung im Zusammenhang mit der Überbürdungsfrage », Heilbronn, Gebr. Henninger, 1888

TIENDREBEOGO, K., Contribution des supports visuels et sonores à l'enrichissement de l'enseignement de l'allemand au secondaire au Burkina Faso en particulier à travers le manuel IHR und WIR. Mémoire de fin de stage de formation aux fonctions d'inspecteur de l'enseignement secondaire, option allemand, 1998.

TRAEGER, B., Das kretische Labyrinth, Rethymnon, Mitos, 2005.

VIËTOR, W., « Der Sprachunterricht muß umkehren ! Ein Beitrag zur Überbürdungsfrage », Heilbronn : Gebr. Henninger, 1882.

\section{NOTES}

1. L'acquisition de cette compétence concerne aussi les apprenants des autres langues étrangères telles que l'anglais, le chinois ou le français langue seconde au Burkina Faso (cf. Bationo, $2010 \mathrm{c}$ ).

2. Le concept de communication est à comprendre ici comme un échange réciproque d'idées, d'opinions, etc. entre, au moins, deux interlocuteurs qui utilisent la langue et parfois la gestuelle. 3. Traduit par nous.

4. La conversation littéraire est conçue par Christ et al. (1993 : 25) comme un échange, voire une discussion ou un entretien, entre apprenants et enseignants sur un thème en cours de littérature. 
5. Par compétence communicationnelle, nous entendons la compétence de communication, c'està-dire de l'habileté à communiquer avec un interlocuteur de langue étrangère en tenant compte de la culture de celui-ci (cf. Kast, 1985 : 4). Selon Allhoff (2001:9), c'est la capacité d'échanger, voire de parler les uns avec les autres.

6. Même si l'enquête qui a été menée en 2004 n'avait pas pour objet de renseigner sur le niveau des apprenants conformément à l'échelle du CECR, elle a permis tout de même de savoir qu'apprenants et enseignants éprouvent le besoin d'améliorer le niveau en expression orale et écrite. L'échelle du CECR permettra de poursuivre cette réflexion dans d'autres travaux, car des recherches n'ont pas encore été menées sur le niveau réel des apprenants d'allemand selon l'échelle du CECR : A1, A2, B1, B2, C1, C2 (op.cit. pp. 60-61).

7. Pour plus de détails sur ces résultats, voir Bationo, 2007 a.

8. Au Cameroun par exemple, $39,21 \%$ des enseignants d'allemand ne sont pas satisfaits de la prestation orale de leurs élèves (cf. Ngatcha, 1991 : 51). En outre, nous reconnaissons avec Dolz et Schneuwly (1998: 11) que les difficultés en expression orale concernent également les utilisateurs des autres langues telles que le français langue maternelle ou langue étrangère et même le patois, d'où la nécessité de l'enseigner.

9. Pour plus d'informations sur ces résultats, voir Bationo, 2007 a : 65.

10. Traduction du texte original effectuée par nos soins : Das Ziel des Unterrichts in den neueren Sprachen hat in allen höheren Schulen eine Änderung dahin erfahren, daß der praktische mündliche und schriftliche Gebrauch der Fremdsprache [...] überall in den Vordergrund gestellt, die Grammatik nur Mittel zum Zwecke ist.

11. Pour plus de détails sur le rapport littérature et labyrinthe, se référer à Humière, 2009 : 133-144; Message, 2009 : 189-201 et Traeger, 2005.

12. Les auteurs suivants peuvent être cités à titre d'exemple : Jean-Félix Belinger : Ngono Mefane das Mädchen der Wälder; Daniel Mépin : Die Weissagung der Ahnen, Tonè Tonè ; Yomb May : Die BasaaSagen; Philomène Atyamé: Abengs Entscheidung, etc. Pour plus de détails, voir l'anthologie littéraire interculturelle de Bationo, $2007 \mathrm{~b}$.

\section{RÉSUMÉS}

La première fonction de toute langue est la communication. On peut soutenir alors que l'enseignement de l'allemand en tant que langue étrangère au Burkina Faso poursuit comme objectif fondamental le développement chez les apprenants de la capacité à communiquer tant à l'oral qu'à l'écrit. Si on entend par littérature, polysémie, esthétique et fiction, on se pose alors la question de savoir dans quelle mesure elle pourrait jouer un rôle dans l'acquisition de la langue allemande. La présente réflexion montre que la littérature a des potentialités linguistiques et culturelles susceptibles de contribuer à l'acquisition de la compétence communicationnelle des apprenants d'allemand au Burkina Faso.

The first function of every language is communication. We can support the thesis that teaching German as a foreign language in Burkina Faso pursues as a fundamental objective the development of the learners' ability to communicate orally and in writing. More exactly, because each communication is an interactive process between interlocutors, we expect that the assimilation or the use of the German language enables the learners to understand, to exchange ideas, to share their visions of the world. In this way the German language like any language, 
when it is practised, is an establisher of a community. If on the one hand we define literature as the representation of a created world or an invented existence and on the other hand as imagination, ambiguity and aesthetics or as original creation, constituted by words and ideas, we can then ask the question to know in which measure it could play a role in the process of the learning and the teaching of German as a foreign language. The question is to know if literature facilitates and optimizes the communicative skill. Through the present reflection, we try to answer this question.

\section{INDEX}

Keywords : teaching/learning of German, foreign languages, communicative competence, literature, literary conversation, Burkina Faso

Mots-clés : enseignement/apprentissage de l'allemand, langues étrangères, compétence communicationnelle, littérature, conversation littéraire

\section{AUTEUR}

\section{JEAN-CLAUDE BATIONO}

Université de Koudougou - ENS 